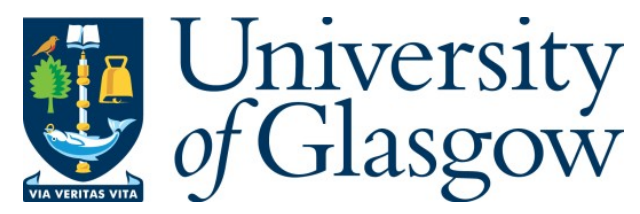

Annese, V. F. , Giagkoulovits, C. , Hu, C. , Al-Rawhani, M. A., Grant, J. , Patil, S. and Cumming, D. (2022) Micromolar metabolite measurement in an electronically multiplexed format. IEEE Transactions on Biomedical Engineering. (Early Online Publication)

(doi: 10.1109/TBME.2022.3147855)

This is the Author Accepted Manuscript.

There may be differences between this version and the published version. You are advised to consult the publisher's version if you wish to cite from it.

http://eprints.gla.ac.uk/263743/

Deposited on: 28 January 2022

Enlighten - Research publications by members of the University of Glasgow http://eprints.gla.ac.uk 


\title{
Micromolar Metabolite Measurement in an Electronically Multiplexed Format
}

\author{
V.F. Annese, C. Giagkoulovits, C. Hu, M.A. Al-Rawhani, J. Grant, S.B. Patil, \\ D.R.S. Cumming
}

\begin{abstract}
Objective: The detection of metabolites such as choline in blood are important in clinical care for patients with cancer and cardiovascular disease. Choline is only present in human blood at low concentrations hence accurate measurement in an affordable point-ofcare format is extremely challenging. Although complementary metal-oxide semiconductor (CMOS) and microfluidics are individually mature technologies, their integration has presented challenges that we overcome in a novel, cost-effective, single-step process.
\end{abstract}

Methods: To demonstrate the process, we present the microfluidic integration of a metabolomics-on-CMOS point-of-care platform with four capillary microfluidic channels on top of a CMOS optical sensor array.

Results: The fabricated device was characterised to verify the required structural profile, mechanical strength, optical spectra, and fluid flow. As a proof of concept, we used the device for the in-vitro quantification of choline in human blood plasma with a limit of detection of $3.2 \mu \mathrm{M}$ and a resolution of $1.6 \mu \mathrm{M}$.

Significance: Integration of microfluidics on to CMOS technology has the potential to enable advanced sensing technologies with extremely low limit of detection that are well suited to multiple clinical metabolite measurements.

Index Terms- CMOS, microfluidics, point-of-care, mobile technology, lab-on-chip, metabolomics, microfabrication, sensor array, microarrays, system integration.

\section{INTRODUCTION}

More-than-Moore complementary metal-oxide semiconductor (CMOS) technology has the potential to extend the capability and exploitation of microelectronics into new and emerging markets such as wireless point-of-care (POC) diagnostics [1]. Hybrid microfluidic-CMOS systems offer unique advantages for delivering high-throughput, rapid and affordable technology for diagnostic measurements made using bodily fluids such as blood and urine. Microfluidics provides an efficient approach to reducing reagents and sample volume and enables multiplexing of biological assays [2]. Integrated sensors and circuits have high accuracy, low power

'V.F.A., C.G., C.H., M.A.A., J.G., S.B.P., D.R.S.C. are with the James Watt School of Engineering, University of Glasgow, Glasgow, G12 8QQ, UK (e-mail: david.cumming.2@glasgow.ac.uk). consumption, low cost and are ideal for portable and miniaturized diagnostic systems. As an electronic component, CMOS is well-suited to enhanced functionality of wireless technologies such as smartphones and tablets to create fast and accurate multi-analyte POC diagnostic devices. Testing multiple biomarkers enables pattern recognition strategies which can improve diagnostic accuracy and detect early onset of deadly diseases $[3,4]$.

A further benefit of microfluidic-CMOS integration is the potential for improved limit of detection (LOD) when measuring small samples, exploiting close proximity and reduced interference that would otherwise impact the signalto-noise ratio of a system [5]. Low LOD CMOS-based devices have been reported with no multiplexing capability [6, 7]. Wash steps are frequently needed. Furthermore, a hybrid microfluidic-CMOS system has the potential to enable a new generation of simple to use optical POC technologies measuring multiple blood biomarkers in micro- and nanomolar concentrations. For example, there have been extensive studies in digital polymerase chain reaction (PCR) technology [8], portable nucleic acid quantitative detection devices [9] and fully integrated nucleic acid detection platforms [10]. A hybrid microfluidic-CMOS system has the potential to combine genomics with other omics technology, including metabolomics and proteomics, leading to a new era of multiomics POC platforms.

Many fabrication techniques have been proposed in the literature for delivering hybrid microsystems, including micromachining [11, 12], laser ablation [13, 14], replica moulding $[15,16]$ and printing [17]. Table I shows the prior art in the field. A recurring problem is the scale mismatch between microelectronic and microfluidic devices. A CMOS chip's cost is proportional to its area, so integrated circuit technology is driven to be small (Moore's Law). Although microfluidic channel sizes are compatible with CMOS elements, typically fluidic input/output (I/O) ports need to be large enough (hundreds of micrometers) to allow for a practical interconnect and fluid sampling. Thus, using a small CMOS chip to reduce the cost, the fluidic area is usually extended beyond the chip's perimeter by embedding it in a planarized surrounding medium $[11,14,18]$. The packaging and planarization that is required entails additional processing steps that complicate the manufacturing and increase the cost of the final device. 
TABLE I

INTEGRATION OF INTEGRATED CIRCUITS (IC) WITH MICROFLUIDICS.

\begin{tabular}{|c|c|c|c|c|c|c|}
\hline 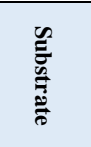 & $\frac{\rho}{\partial \frac{2}{2}}$ & 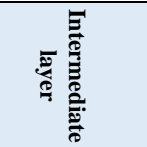 & 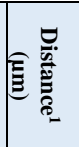 & 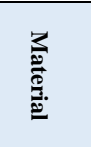 & $\begin{array}{l}\overrightarrow{0} \\
\overrightarrow{\tilde{E}} \\
\text { है. }\end{array}$ & $\underset{0}{\overparen{0}}$ \\
\hline CMOS & - & Yes & 300 & $\begin{array}{l}\text { SU-8 } \\
\text { glass }\end{array}$ & $\begin{array}{l}\text { Planarization } \\
\text { Photolithography }\end{array}$ & [11] \\
\hline $\begin{array}{l}\mathrm{IC} / \\
\text { flexible } \\
\text { PCB }\end{array}$ & - & $\begin{array}{l}\text { Yes } \\
\text { (PDMS) }\end{array}$ & 120 & glass & Laser engrave & [13] \\
\hline $\begin{array}{l}\text { CMOS/ } \\
\text { flexible } \\
\text { PCB }\end{array}$ & 4 & $\begin{array}{l}\text { Yes } \\
\text { (Polyimide) }\end{array}$ & 85 & PDMS & $\begin{array}{l}\text { Replica moulding } \\
\text { Adhesive bonding }\end{array}$ & {$[15]$} \\
\hline CMOS & - & $\begin{array}{l}\begin{array}{l}\text { Yes } \\
\text { (photoresist) }\end{array} \\
\text { (pol }\end{array}$ & 1.8 & PMMA & $\begin{array}{l}\text { Planarization } \\
\text { Laser engrave }\end{array}$ & [14] \\
\hline IC & 1 & No & 0 & PDMS & $\begin{array}{l}\text { Replica moulding } \\
\text { Plasma bonding }\end{array}$ & [16] \\
\hline CMOS & 1 & No & 0 & Epoxy & Direct writing & {$[17]$} \\
\hline CMOS & - & No & 0 & SU-8 & $\begin{array}{l}\text { Photolithography } \\
\text { Plasma bonding }\end{array}$ & [12] \\
\hline CMOS & 1 & No & 0 & $\begin{array}{l}\text { SU-8 } \\
\text { PDMS }\end{array}$ & $\begin{array}{l}\text { Planarization } \\
\text { Photolithography } \\
\text { Plasma bonding }\end{array}$ & [18] \\
\hline CMOS & - & No & 0 & PDMS & $\begin{array}{l}\text { Replica moulding } \\
\text { Encapsulation }\end{array}$ & [20] \\
\hline CMOS & 4 & No & $\mathbf{0}$ & $\begin{array}{l}\text { Epoxy } \\
\text { PDMS }\end{array}$ & $\begin{array}{l}\text { Replica moulding } \\
\text { Injection moulding } \\
\text { Plasma bonding }\end{array}$ & $\begin{array}{l}\text { This } \\
\text { Work }\end{array}$ \\
\hline
\end{tabular}

Further challenges for integrating microfluidics and CMOS are process compatibility, the topology of the chip, alignment and functionalization [5, 19]. CMOS chips are frequently packaged using wire-bonding. Bonds require encapsulation to minimize breakages, owing to their fragile nature, and for electrical insulation. Unlike traditional electronic packaging, fluidic packaging has not been standardized. Thus, the approach to accommodate fluidics on CMOS is either to modify a pre-existing standard package or develop a custom one. Alternative methods for hybrid system packaging have been demonstrated including liquid interconnections [20] or flexible printed circuit board (PCB) [15], but they have practical limitations and are not easily reproduced. Current solutions are typically customized, empiric and involve complicated fluidic designs which can be difficult to be used in practical applications. As a consequence of these barriers there is a lack of standardization, reliability and ease of use [21].

In this paper, we present a versatile, reproducible, and affordable process to monolithically integrate passive capillary microchannels with CMOS that eliminates the complexities needed in previous techniques. The process is suitable for manufacturing disposable CMOS cartridges capable of performing multiple measurements at the same time. The technique addresses the technological challenges by using a combination of replica and injection moulding that minimise the production time by reducing the number of steps. The process exploits the epoxy-moulding compound (EMC) IC encapsulation packaging method and benefits from EMC's well-known wire-bond mechanical stabilization properties [22]. As a proof-of-concept, complete devices were demonstrated by the integration of four microfluidic channels on top of a CMOS optical sensor array. The apparatus was used for the rapid quantification of the blood-borne metabolite choline in human plasma (physiological range $7-20 \mu \mathrm{M}$ [23]). Elevated concentrations of choline have been associated with several conditions including cardiovascular disease [24] and cancer [25]. We demonstrate that the hybrid device can perform multiple measurements with a LOD of $3.2 \mu \mathrm{M}$ and resolution of $1.6 \mu \mathrm{M}$. The process is therefore suitable for delivering rapid and affordable POC technology for multiplexed measurements with micromolar LOD.

\section{Design And Simulations}

To demonstrate the proposed integration method, a microfluidic network with four identical microfluidic channels was designed and monolithically integrated on to a custom CMOS sensor array. The device was designed to make measurements of metabolite concentrations in blood products. As a proof of concept, we focused on blood choline. Choline quantification is typically measured with liquid chromatography-tandem mass spectrometry (LC/MS) [26]. Despite the high precision (relative standard deviation: $0.7 \%$ ) and ultra-low LOD (53.6 pM), LC/MS choline measurements require a bulky and specialized equipment and is time consuming and expensive [27]. Choline quantification has also been performed with biosensing techniques, including absorbance, fluorescence, chemiluminescence and electrochemistry [26]. Choline biosensors with LOD in the micromolar range have been shown in the literature [26]. However, usually they can measure only a single metabolite and do not have pre-loaded dried reagents. They therefore have practical limitations inhibiting their deployment.

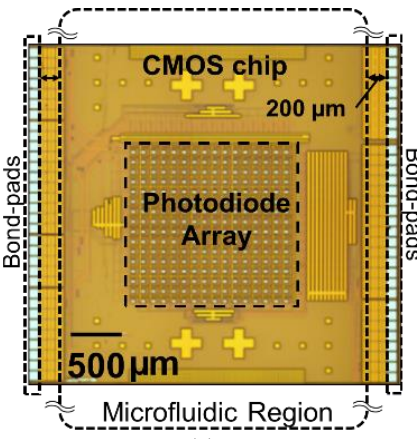

(a)

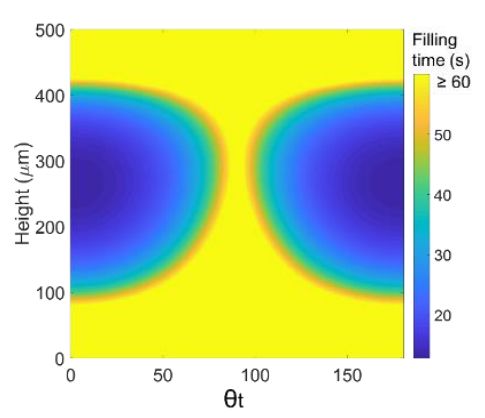

(b)

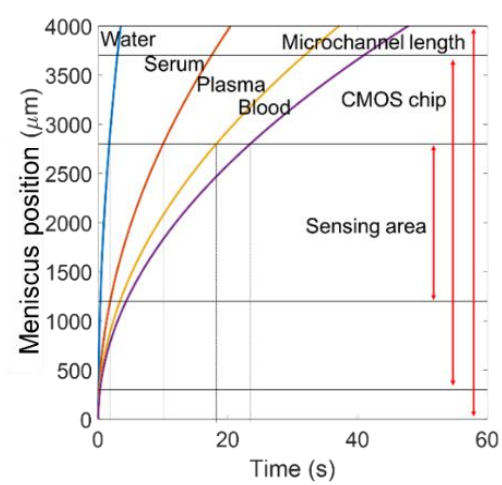

(c)

Fig. 1. (a) CMOS sensor array. (b) Simulations of the filling time of a microfluidic channel for different combinations of $h$ and $\theta_{t}$ for blood (w = $300 \mu \mathrm{m} ; \theta_{s}=98.4^{\circ} ; \theta_{b}=78.2^{\circ} ; L=4 \mathrm{~mm}$ ). (c) Simulations of the meniscus position with respect to the inlet in a microchannel with $\mathrm{w}=h=300 \mu \mathrm{m} ; \theta_{s}=98.4^{\circ} ; \theta_{b}=78.2^{\circ} ; \theta_{t}=32.5^{\circ} ; L=4 \mathrm{~mm}$. 
TABLE II

SIMULATION PARAMETERS

\begin{tabular}{|c|c|c|c|}
\hline \multicolumn{2}{|l|}{ Design Equation } & \multicolumn{2}{|c|}{ Relation } \\
\hline \multicolumn{2}{|l|}{ Hagen-Poiseuille } & \multicolumn{2}{|c|}{$\Delta \mathrm{p}=\mathrm{R}_{\mathrm{h}} \mathrm{Q}$} \\
\hline \multicolumn{2}{|c|}{$\begin{array}{l}\text { Hydraulic resistance } \\
\text { (rectangular cross-section) [29] }\end{array}$} & \multicolumn{2}{|c|}{$\mathrm{R}_{\mathrm{h}}=\frac{12 \eta \mathrm{L}}{\left\{1-0.63\left(\frac{\mathrm{h}}{\mathrm{w}}\right)\right\} \mathrm{h}^{\mathrm{a}} \mathrm{w}}$} \\
\hline \multicolumn{2}{|l|}{ Meniscus position } & \multicolumn{2}{|c|}{$\mathrm{l}(\mathrm{t})=\mathrm{h} \sqrt{\frac{\Delta \mathrm{p}}{6 \eta \mathrm{L}}\left(1-0.63 \frac{\mathrm{h}}{\mathrm{w}}\right) \mathrm{t}}$} \\
\hline \multicolumn{2}{|l|}{ Capillary pressure } & \multicolumn{2}{|c|}{$\Delta \mathrm{p}=\gamma\left(\frac{\cos \theta_{\mathrm{b}}+\cos \theta_{\mathrm{t}}}{\mathrm{h}}+\frac{2 \cos \theta_{\mathrm{s}}}{\mathrm{w}}\right)$} \\
\hline Fluid/Material & \multicolumn{3}{|c|}{ Design Parameter } \\
\hline Water & \multicolumn{2}{|c|}{$\eta=0.84[\mathrm{mPa} / \mathrm{s}][33]$} & $\gamma=0.073[\mathrm{~N} / \mathrm{m}][33]$ \\
\hline Human Serum & \multicolumn{2}{|c|}{$\eta=1.4[\mathrm{mPa} / \mathrm{s}][34]$} & $\gamma=0.050[\mathrm{~N} / \mathrm{m}][35]$ \\
\hline Human Plasma & \multicolumn{2}{|c|}{$\eta=1.7[\mathrm{mPa} / \mathrm{s}][34]$} & $\gamma=0.045[\mathrm{~N} / \mathrm{m}][35]$ \\
\hline Human Blood & \multicolumn{2}{|c|}{$\eta=2.4[\mathrm{mPa} / \mathrm{s}][34]$} & $\gamma=0.056[\mathrm{~N} / \mathrm{m}][36]$ \\
\hline PDMS & \multicolumn{3}{|c|}{$\theta_{t}=107^{\circ}$ (measured); $100^{\circ}-110^{\circ}[37]$} \\
\hline Plasma-treated PDMS & \multicolumn{3}{|c|}{$\theta_{\mathrm{t}}=<10^{\circ}[37]$} \\
\hline PVA-coated PDMS & \multicolumn{3}{|c|}{$\theta_{t}=32.5^{\circ}$ (measured); $20^{\circ}-40^{\circ}[37]$} \\
\hline Epoxy resin & \multicolumn{3}{|c|}{$\theta_{s}=98.4^{\circ}$ (measured); $75^{\circ}-100^{\circ}[38]$} \\
\hline CMOS Chip & \multicolumn{3}{|c|}{$\theta_{b}=78.2^{\circ}$ (measured) } \\
\hline \multicolumn{4}{|c|}{$\begin{array}{l}\text { Notations: } \triangle p: \text { Pressure gradient; } Q: \text { flow rate; } \eta: \text { dynamic viscosity; } \gamma \text { : } \\
\text { surface tension; } L: \text { channel length; } h \text { : channel height; } w: \text { channel width; } \theta_{b} \text { : } \\
\text { contact angle of bottom material; } \theta_{t}: \text { contact angle of top material; } \theta_{s} \text { : } \\
\text { contact angle of side material. }\end{array}$} \\
\hline
\end{tabular}

The CMOS sensor array used in this work was a custom $16 \times 16$ array of sensing elements (Fig. 1(a)). Each element was $100 \mu \mathrm{m} \times 100 \mu \mathrm{m}$ and integrated a photodiode sensor. The size of the chip was $3.4 \mathrm{~mm} \times 3.6 \mathrm{~mm}$. The CMOS chip has been fully characterized in previous works [28]-[31]. The chip required wire bonding for use. By having bond-pads to the left and right-hand sides only, a fluidic region was left clear for microchannels to run from top to bottom with no interruption from bond wires (see Fig. 1(a)). Fluidic I/O were places at the top and bottom edges of the chip.

To perform a choline test, we use an enzyme-based colorimetric assay. We introduce an assay reagent formulation into the channels that uses a metabolite-specific enzyme, choline oxidase $(\mathrm{CHOX})$, that liberates $\mathrm{H}_{2} \mathrm{O}_{2}$ during the reaction with choline. The $\mathrm{H}_{2} \mathrm{O}_{2}$ in turn reacts with peroxidase (HRP), phenol and 4-aminoantipyrine (4-AAP) to produce a colorimetric change that increases the optical absorbance of the liquid in the channels that is detected by the on-chip photodiodes. More details are given in Section III. The reaction is measured as a function of time. Thus, the concentration of the metabolite can be calculated using the Michaelis-Menten equation by determining the initial reaction rate [28]:

$$
\frac{\mathrm{d}[\mathrm{P}]}{\mathrm{dt}}=\frac{V_{m} \cdot[\mathrm{S}]}{K_{m}+[\mathrm{S}]}
$$

where $[\mathrm{P}]$ and $[\mathrm{S}]$ are the concentrations of the reaction product and the substrate, $V_{m}$ is the maximum rate achieved by the system and $K_{m}$ is the Michaelis constant.

It is desirable that sensing area of the device should be covered rapidly by the fluid flowing in the microfluidic channel (i.e. filling time) to obtain an accurate estimation of the initial reaction rate. In order to choose a design that would permit fast filling of the channels, the filling time of passive microfluidic channels was modelled in Matlab using the fluid mechanics equations shown in Table II [32]. The microchannels were designed with a rectangular section of width, $w$; height, $h$; and length, $L$. The effect of contact angle was modelled using water as an initial approximation to the blood products in use. The contact angles to the bottom, top and side materials, denoted $\theta_{b}, \theta_{t}$, and $\theta_{s}$ respectively, were introduced to the model since different materials were used.

Simulations to analyse the fluid flow in the microstructure with water, human serum, plasma, blood were performed with appropriate values for the dynamic viscosity, $\eta$, and the surface tension $\gamma$. Table II summarises the parameters used. The effect of $h$ and $\theta_{t}$ on the filling time for the example of whole blood is shown in Fig. 1(b). Simulations indicated that a microfluidic channel made with $w=300 \mu \mathrm{m}, h=300 \mu \mathrm{m}$, $L=4 \mathrm{~mm}, \theta_{b}=78.2^{\circ}, \theta_{s}=98.4^{\circ}$, and $\theta_{t}=32.5^{\circ}$ minimised the filling time. The simulated filling time for water, serum, plasma and blood in the designed microstructure were found to be $1.7 \mathrm{~s}, 10.6 \mathrm{~s}, 18.1 \mathrm{~s}$, and $23.3 \mathrm{~s}$, respectively (see Fig. 1(c)). Using these simulations, a photomask for lithography was designed using Tanner L-EDIT from Mentor Graphics.

\section{FABRICATION}

The proposed integration method uses a combination of replica and injection moulding and is illustrated in Fig. 2.

A SU-8 mould was fabricated using a photolithographic process on a 4" silicon wafer. The target microchannel height was higher than the typical SU-8 3050 thickness hence a double layer of photoresist was necessary. SU-8 3050 was spin-coated on the wafer for $30 \mathrm{~s}$ at $1000 \mathrm{rpm}$ and then sequentially baked for $1 \mathrm{~min}$ at $65^{\circ} \mathrm{C}, 90 \mathrm{~min}$ at $90^{\circ} \mathrm{C}$ and $1 \mathrm{~min}$ at $65^{\circ} \mathrm{C}$ on a vacuum hotplate. A second layer of SU-8 3050 was then spin-coated on to the wafer and baked as before (Fig. 2(a)). The sample was patterned by exposing it twice to ultraviolet light with a $15 \mathrm{~s}$ waiting time between exposures using a Karl Suss mask aligner (MA6). Each exposure was for $70 \mathrm{~s}$ (Fig. 2(b)). The sample was then baked on a vacuum hotplate for $2 \mathrm{~min}$ at $65^{\circ} \mathrm{C}, 10 \mathrm{~min}$ at $90{ }^{\circ} \mathrm{C}$ and $2 \mathrm{~min}$ at $65^{\circ} \mathrm{C}$. The patterns were developed using ethylene carbonate solvent (development time $28 \mathrm{~min}$ ). The wafer was then rinsed with isopropyl alcohol (IPA) and baked (30 min at $180{ }^{\circ} \mathrm{C}$ ) (Fig. 2(c)).

The SU-8 mould was silanised by exposure for $30 \mathrm{~min}$ to Trichloro $(1 \mathrm{H}, 1 \mathrm{H}, 2 \mathrm{H}, 2 \mathrm{H}$-perfluorooctyl) silane in a vacuum chamber (Fig. 2(d)). The silanisation process aided the later removal of PDMS from the SU-8 mould. The wafer was then placed into a petri dish. $25 \mathrm{~g}$ of a $1: 14 \mathrm{w} / \mathrm{w}$ mixture of PDMS:curing agent was poured onto the mould and degassed for 1 hour in a vacuum chamber (Fig. 2(e)). The PDMS was then cured $\left(2 \mathrm{~h}\right.$ at $\left.70{ }^{\circ} \mathrm{C}\right)$, released from the SU-8 mould, placed on to a clean substrate, and cut with a sharp knife (Fig. 2(f)). The PDMS microstructure was then temporarily bonded to the CMOS chip using a flip-chip bonder (Model 850, Semiconductor Equipment Corporation) (Fig. 2(g)). Patterns that were present on both the chip and the PDMS 
microstructure enabled alignment. There was no oxygen plasma exposure or adhesive coating step before PDMS bonding. The bonding was thus only temporary, as a consequence of Van der Waals forces, and the PDMS mould could later be easily peeled off from the chip using tweezers. The 1:14 mixture of PDMS:curing agent showed better adhesion to the CMOS than the more commonly used 1:10 ratio [39].

The CMOS chip with the PDMS microstructure was bonded to a 120-pin ceramic pin grid array (CPGA) package, purchased from Spectrum Semiconductor Materials, using the EPO-TEK H74 epoxy from Epoxy Technology Inc (Fig. 2(h)). The overall size of the selected CPGA package was $3.3 \mathrm{~cm} \times 3.3 \mathrm{~cm}$, with a $1 \mathrm{~cm} \times 1 \mathrm{~cm}$ cavity accommodating the structure to be wire-bonded. The CMOS chip was then wire-bonded to the CPGA package using the Hesse and Knipps Bondjet 710 (Fig. 2(i)).

Black epoxy resin (302-3M 1LB by Epoxy Technology Inc.) was flowed into the PDMS microstructure (Fig. 2(j)). The PDMS structure was designed to be larger than the chip area in the top and bottom directions (Fig. 2(k)). As such, in the same epoxy-moulding processing step, we were able to perform wire-bond encapsulation (left and right sides, see Fig. 2(1)), planarization (top and bottom of chip, see Fig. 2(m)) and injection moulding for microfluidic fabrication. After the epoxy resin was cured ( $48 \mathrm{~h}$ at room temperature), the PDMS structure was removed from the CMOS chip, leaving behind the open epoxy microchannels (Fig. 2(n)). The open channels can then be functionalized for a specific assay (Fig. 2(o)). For the colorimetric determination of choline, a reaction mixture composed of $6.7 \mu \mathrm{L}$ CHOX $(150 \mathrm{U} / \mathrm{mL}), 6.7 \mu \mathrm{L}$ HRP (300U/ml), $3.3 \mu \mathrm{L}$ phenol $(44.5 \mathrm{mM})$, and $3.3 \mu \mathrm{L}$ 4-AAP $(10.5$ $\mathrm{mM}$ ) was prepared.

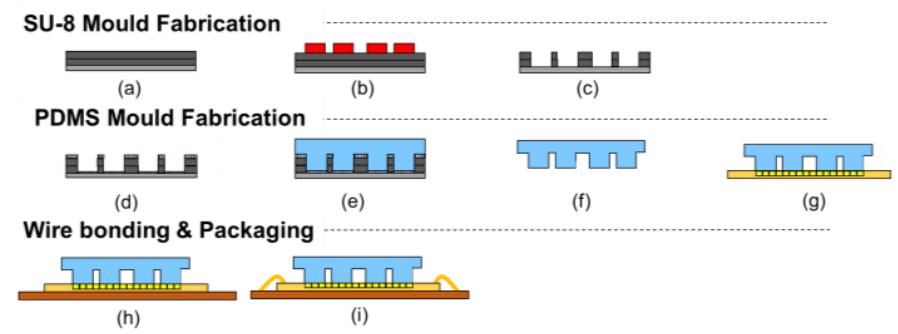

Epoxy microstructure, functionalisation and channel enclosure

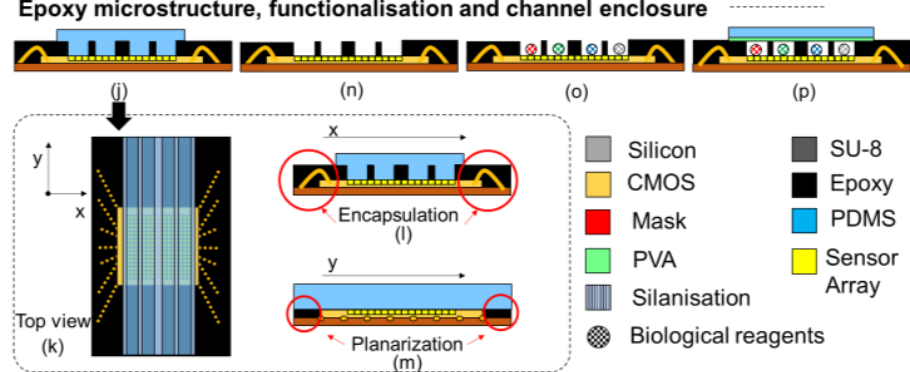

Fig. 2. (a) SU-8 3050 coating; (b) Photolithography; (c) SU-8 mould; (d) Silanisation; (e) PDMS casting; (f) PDMS microstructure; (g) PDMS microstructure temporarily bonded onto the CMOS; (h) CMOS chip bonded onto a ceramic package; (i) Wire-bonding; (j) Epoxy injection; (k) Top view; (I) Injected epoxy encapsulate the wire-bonds and (m) planarizes the surface; ( $n$ ) PDMS is removed after epoxy is cured; (o) Functionalisation; (p) Microchannel enclosure with PVA-coated PDMS.
All chemicals were purchased from Sigma Aldrich and prepared in DI water. One microliter of the reaction mixture was pipetted in each microfluidic channel using ultralong microloader pipette tips (Eppendorf). The device was then dried at room temperature in a vacuum chamber for 1 hour, leaving the pre-loaded reagents in a dried state.

To enclose the microchannels, a plain slab of PDMS was cut with a sharp knife $(4 \mathrm{~mm} \times 3 \mathrm{~mm})$, cleaned, exposed to oxygen plasma $(1 \mathrm{~min}$ at $80 \mathrm{~W})$ and immersed in a water based polyvinyl acetate (PVA) solution (1\% w/w , $10 \mathrm{~min})$ to achieve a uniform PVA coating [37]. The surface of the PVA-coated PDMS was activated using oxygen plasma $(80 \mathrm{~W}$ for $45 \mathrm{~s}$ ) and then brought in contact with the epoxy open channel under constant pressure of 5 psi for 15 min, creating enclosed microchannels (Fig. 2(p)). Devices fabricated without the functionalization step underwent an additional baking step $\left(15 \mathrm{~min}\right.$ at $\left.90{ }^{\circ} \mathrm{C}\right)$.

\section{Results}

\section{A. Fabrication Results}

Microchannels were successfully fabricated using the design specifications. Micrographs of the resulting SU-8 mould, PDMS microstructure and final device are shown in Fig. $3(\mathrm{a}-\mathrm{c})$. The misalignment of the microchannel integration process, evaluated over three different samples, was estimated to be less than $25 \mu \mathrm{m}$ on both axes (Fig. 3(d)).

The height and width of the microstructures with a double SU-8 layer was, on average, $291.95 \pm 6.44 \mu \mathrm{m}$ and $300.87 \pm 0.86 \mu \mathrm{m}$, respectively, as shown in Fig. 3(f, g). The trench had a deep slope of approximately $929 \mu \mathrm{m} / \mu \mathrm{m}$ (Fig. 3(h)). The length of the channel was, on average, $4.0 \pm 0.1 \mathrm{~mm}$.

The mechanical strength of the microfluidic structure was quantified using both tensile and shear testing. Destructive tensile testing was performed using two unfunctionalized devices on a custom setup. An increasing tensile force was applied to the PDMS lid and the chip package to separate them. The PDMS structure was stressed then deformed before peeling off from the substrate. Irreversible PDMS damage started when applying a force of $70 \mathrm{~g}$ - see Fig. 3(i). Shear testing was carried out using a DAGE $4000 \mathrm{HS}$ bond tester on unfunctionalized test structures made from PDMS microchannels bonded to a $3.4 \mathrm{~mm} \times 3.4 \mathrm{~mm}$ silicon substrate that had a $500 \mathrm{~nm}$ coating of silicon nitride. The shear test showed that the bonds could tolerate a maximum lateral force of $576 \mathrm{~g}$ - see Fig. 3(j).

A spectrometer was used to measure the optical transmittance of the materials used for fabricating the device (ffTA-1 from Foster and Freeman, see Fig. 3(k)). For this evaluation, test structures were fabricated onto a glass substrate. The PVA coating was found to slightly reduce the transmittance of the untreated PDMS from 98.8\% to $95.3 \%$ @ $500 \mathrm{~nm}$. The epoxy regions between the channels had a small optical transmittance of $25.28 \%$. The final device is shown in Fig. 3(1). 


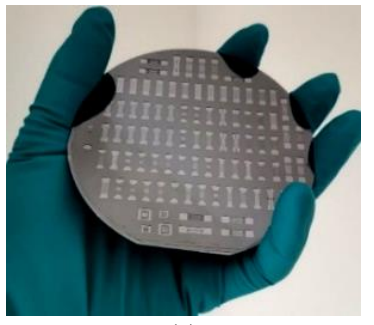

(a)

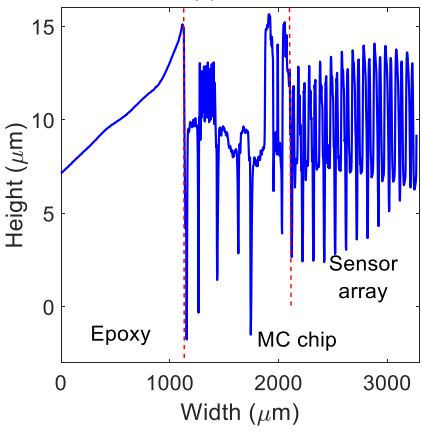

(e)

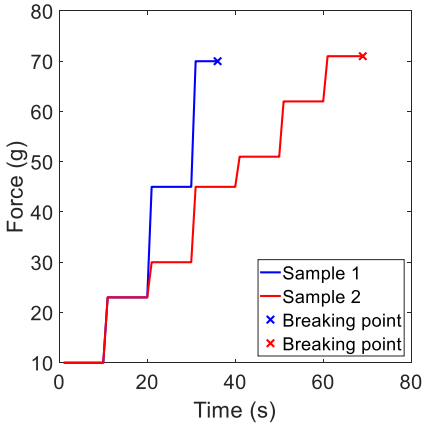

(i)

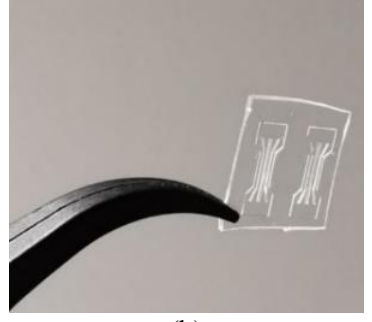

(b)

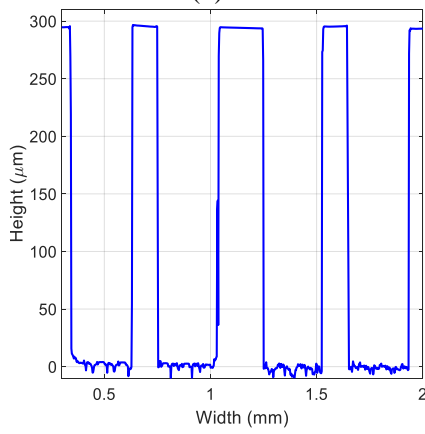

(f)

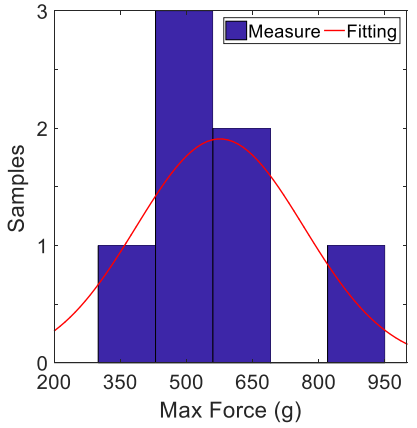

(j)

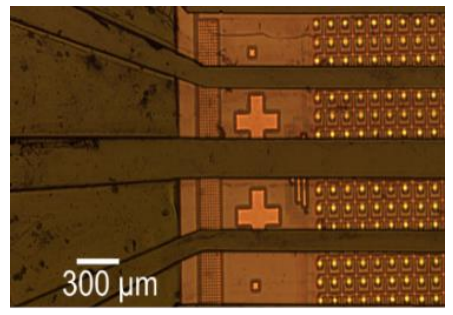

(c)

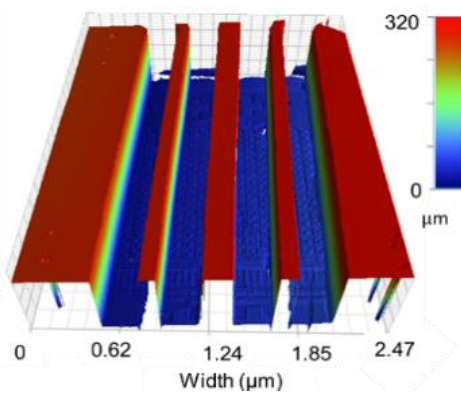

(g)

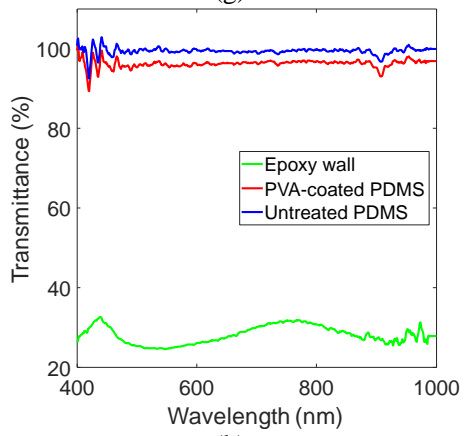

(k)

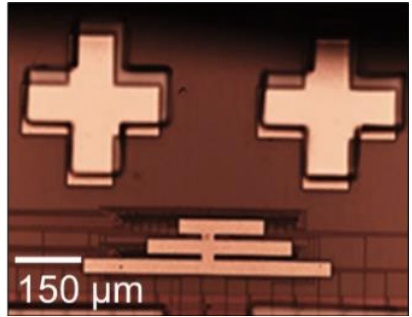

(d)

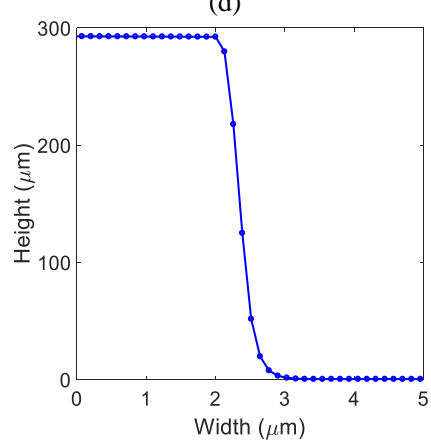

(h)

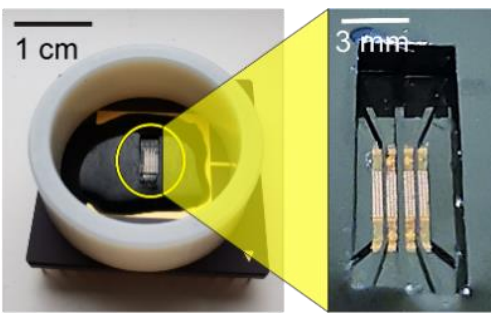

(1)

Fig. 3. (a) SU-8 mould. (b) PDMS mould. (c) Epoxy microfluidics. (d) Misalignment. (e) Surface flatness of the planarized area. (f) Cross-section of a 4-microchannel network. (g) Optical profile. (h) Microfluidic wall. (i) Tensile testing. (j) Shear testing. (k) Optical spectra. (I) Final device.

\section{B. Fluid Flow Characterisation}

The flow rate of the fluids was quantified in unfunctionalized devices using the optical sensor array embedded into the CMOS chip. The device's frame rate of $36.5 \mathrm{fps}$ gave a temporal resolution of $27.4 \mathrm{~ms}$. Capillary action was verified by visual inspection which also showed that the microstructure was successful in drawing the liquid into the microchannel with no leakage to adjacent channels (Fig. 4(a)). The introduction of a sample into the microstructure created a detectable change in the output of the sensors. Photodiodes recorded a voltage spike and a sudden increase of transmittance once each sensor in turn was covered with the added liquid. The signal was a consequence of the decrease of the refractive index contrast producing a reduction of the light reflected from the interface. The spike, presumably produced as the result of the rapid transit of the meniscus which has different optical properties, was very pronounced and clearly detectable, as shown in Fig. 4(b).

Sensors in the same channel and equidistant from the channel inlet responded with simultaneous voltage spikes (Fig. 4(c)). This demonstrated that the meniscus advanced uniformly in the channel. Sensors positioned increasing distance from the channel inlet responded with sequential spikes, effectively recording the advancement of the meniscus (Fig. 4(d)). The flow rate, and the filling time of diluted serum (1:10), diluted plasma (1:1) and whole blood were experimentally measured. Each experiment was repeated three times using the same device. The results were thus calculated as the average and standard deviation over the three identical experiments. Human serum and plasma were purchased from Sigma Aldrich and diluted in DI water. Human blood was purchased from Cambridge Bioscience.

For diluted serum, the time required for the sample to reach the first and the last pixel (filling time) of the array were $2.48 \pm 0.06 \mathrm{~s}$ and $4.58 \pm 0.129 \mathrm{~s}$. The same figures increased to $2.44 \pm 0.01 \mathrm{~s}$ and $7.67 \pm 0.01 \mathrm{~s}$, respectively, for diluted plasma (1:1). The time required for the blood to reach the first and the last pixel of the array were $13.05 \pm 5.20 \mathrm{~s}$ and $28.23 \pm 11.77 \mathrm{~s}$.

Experimental data was compared to simulations with adjusted parameters. The adjusted viscosity and surface tension values of the experimental solutions were obtained using diluted human specimens and estimated using a linear regression. The correlation between experimental and simulated data was $\mathrm{R}^{2}>0.98$ using $w=301 \mu \mathrm{m}, h=292 \mu \mathrm{m}, L=4.0 \mathrm{~mm}$, $\theta_{b}=78.2^{\circ}, \theta_{s}=98.4^{\circ}$, and $\theta_{t}=32.5^{\circ}$ for the simulation (Fig. 4(e)). 


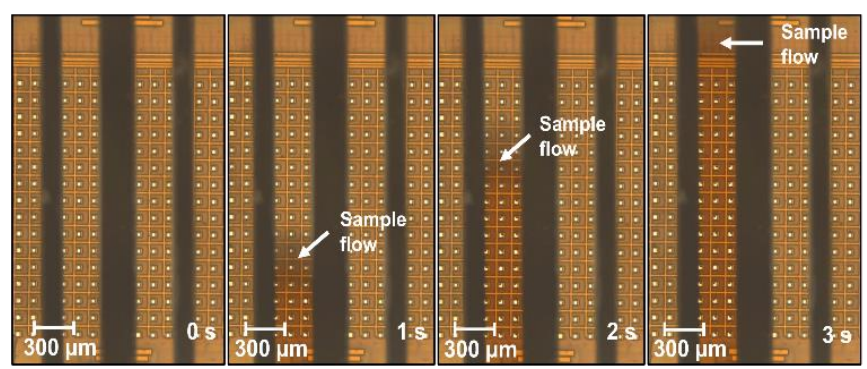

(a)

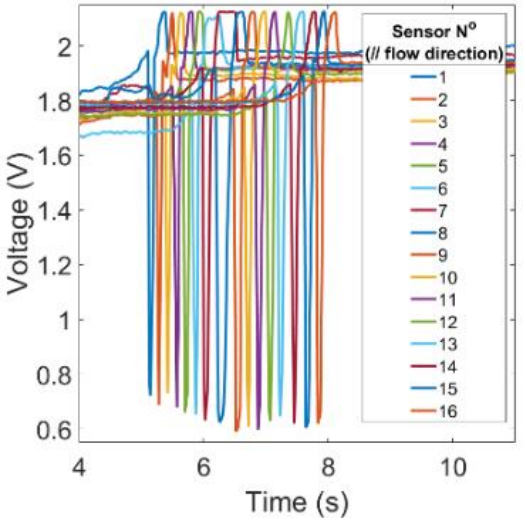

(d)

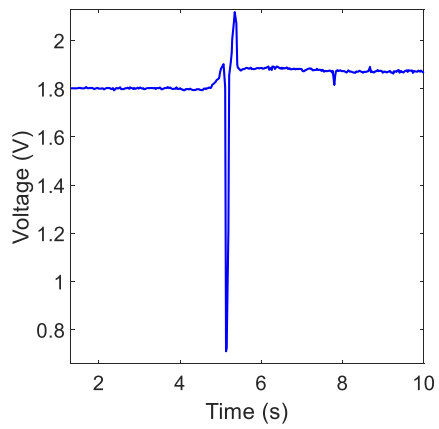

(b)

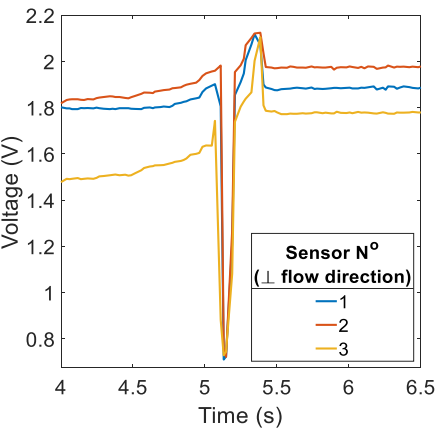

(c)

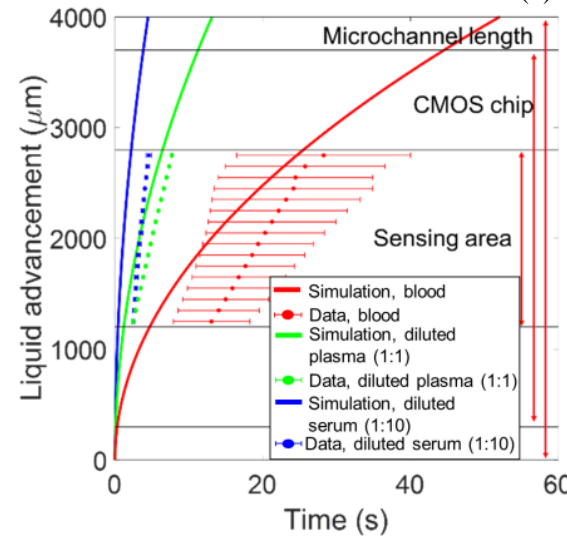

(e)

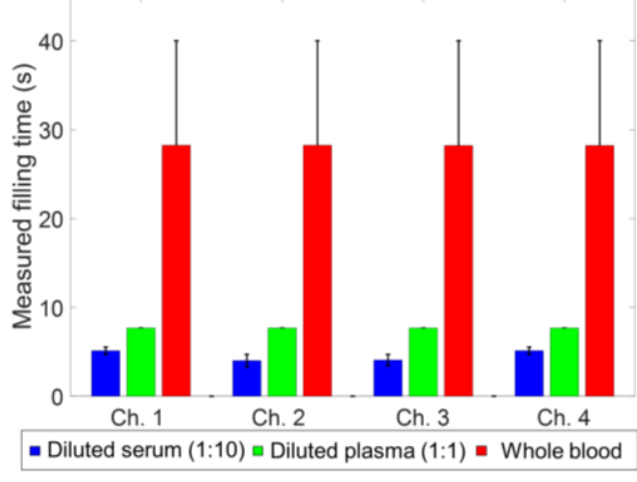

(f)

Fig. 4. (a) Photographs showing the fluid sample (water) covering the sensing area of the CMOS chip in $\sim 3$ seconds. (b) Raw data from the photodiode array. Each photodiode records a sudden voltage spike followed by an increase of the light transmission when the fluid in the channel reaches a sensor area in the channel. (c) In the microchannel, the advancing meniscus simultaneously covered sensors equidistant from the channel inlet. (d) In the microchannel, the advancing meniscus sequentially covered sensors at increasing distance from the channel inlet. (e) Meniscus position with respect to the inlet in the microchannel vs. simulations. (f) Filling time for parallel microfluidic channels using different specimens. Data is reported as the average \pm standard deviation over three replicates for both the subfigures.

The average error between measured data and simulation over all the data points was $3.4 \mathrm{~s}$. The flow of adjacent microchannels, fabricated on the same CMOS chip, was also analysed. This analysis demonstrated that a parallel flow occurred in the microchannels. As expected, the filling time in parallel channels was virtually identical when using the same specimen fluid (see Fig. 4(f)).

\section{Metabolomics on CMOS}

Assays to measure choline using the hybrid microfluidicCMOS device were performed by reading the optical absorbance of the fluid sample at $490 \mathrm{~nm}$ over five minutes. A $3 \mathrm{~mW}$ LED from Thorlabs $(\lambda=490 \mathrm{~nm}, \mathrm{FWHM}=20 \mathrm{~nm}[40])$ with fixed light intensity was used as light source for the absorbance experiments.

The hybrid device was connected to a custom handheld reader using a zero-insertion force socket. The reader was composed of a custom PCB board and a microcontroller (ST Nucleo F334R8 board) programmed before use with custom firmware. The reader was used to interface the sensor array for addressing, data digitization (12 bits, $3.3 \mathrm{~V}$ range) and transmission to a personal computing device (HP EliteBook i7-8650u $16 \mathrm{~GB}$ ) via a USB link. The reader was powered by the USB link $(5 \mathrm{~V})$ that in turn powered the hybrid device (3.3 V). A custom Matlab program was used on the laptop to acquire, visualize, and process data.
To show that microchannels can be independently functionalized, a preliminary experiment was carried out in which only one of the four microchannels was functionalized for a choline assay, as described in Section III. The remaining three channels were functionalized with all other biological reagents except the CHOX enzyme. Choline substrate (choline chloride powder $\geq 98 \%$ ) was purchased from Sigma Aldrich. A test solution containing $250 \mu \mathrm{M}$ choline was prepared in deionized (DI) water. $15 \mu \mathrm{L}$ of testing solution was inserted by pipetting on to the chip's fluidic input. Data collected by the sensors in the same microfluidic channel were averaged [41]. The experiment was repeated three times with the same result. A typical example of the result is presented in Fig. 5(a). The data show that only the channel functionalized with CHOX (Channel 2 in Fig. 5(a)) experienced a detectable signal. The remaining three channels with no CHOX had no response in the presence of choline. No obvious crosstalk between adjacent channels was observed, showing that channels can be independently functionalized. The process has therefore the potential to deliver hybrid devices capable of multiplexed measurements.

To demonstrate choline assay in human plasma, a human plasma sample from a healthy donor was purchased from Cambridge Bioscience. The endogenous choline concentration of the sample was measured by ultra-performance liquid chromatography-tandem mass spectrometry and found to be $14.8 \mu \mathrm{M}$. The sample was then aliquoted. 


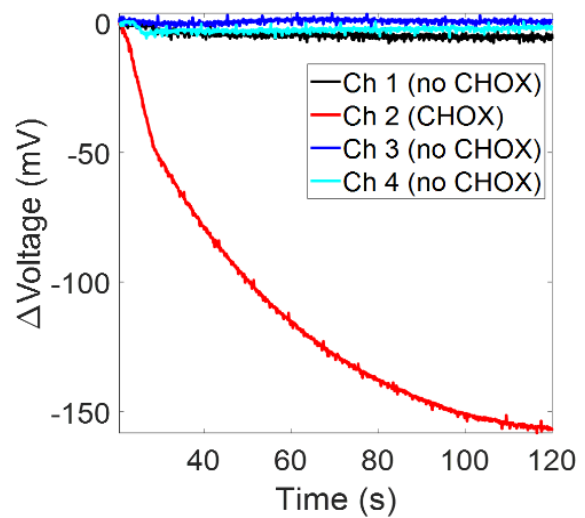

(a)

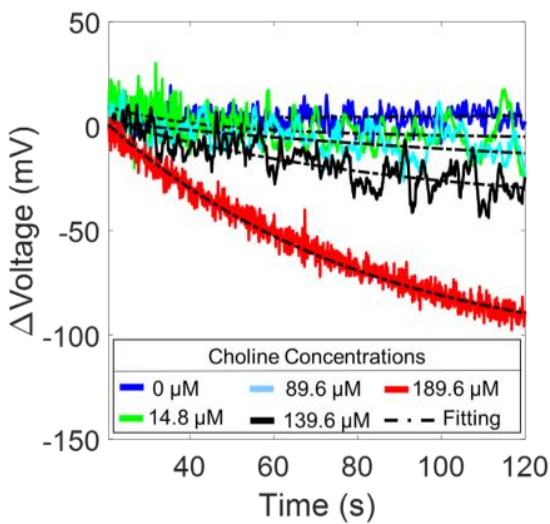

(b)

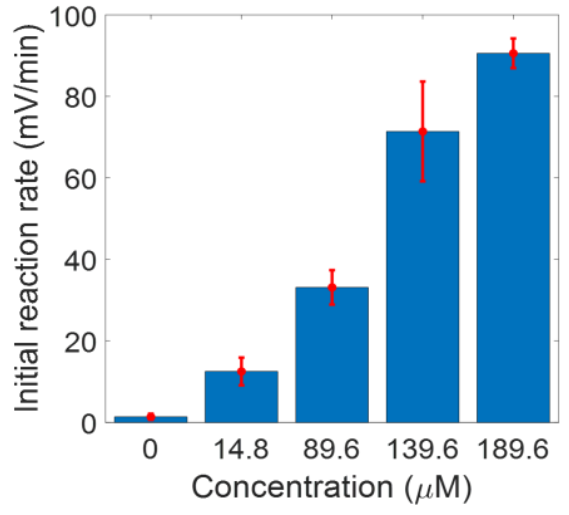

(c)

Fig. 5. (a) Simultaneous measurements of $250 \mu \mathrm{M}$ choline in DI in one functionalised microchannel with $\mathrm{CHOX}$ (Ch 2 ) vs channels with no CHOX (Ch1, Ch3, Ch4). As expected, only the channel functionalised with CHOX shows a detectable response. (b) Measurements of different concentrations of plasma choline in the microchannels (raw data and fitting). (c) Initial reaction rate vs plasma choline concentration.

TABLE III

PLATFORM ChARACTERISATION IN PLASMA FOR CHOLINE.

\begin{tabular}{|c|c|}
\hline Parameter & Value \\
\hline Physiological Range & $7-20 \mu \mathrm{M} \mathrm{[23]}$ \\
\hline Test Range & $0-189.34 \mu \mathrm{M}$ \\
\hline Sample Volume & $15 \mu 1$ \\
\hline Test time & $5 \mathrm{~min}$ \\
\hline Linearity $\left(\mathrm{R}^{2}\right)$ & 0.97 \\
\hline Analytical Sensitivity & $7.78 \cdot 10^{-3} \mathrm{mV} \mathrm{s}^{-1} \mu \mathrm{M}^{-1}$ \\
\hline$V_{m}$ & $162.6 \mathrm{mV} \mathrm{s}^{-1}$ \\
\hline$K_{m}$ & $20.3 \mathrm{mM}$ \\
\hline Precision & $17.7 \%$ \\
\hline Resolution & $1.6 \mu \mathrm{M}$ \\
\hline LOD & $3.2 \mu \mathrm{M}$ \\
\hline
\end{tabular}

Aliquots were modified with additional known concentrations of choline substrate. For each test concentration of choline, the assay was performed using all four channels simultaneously functionalized for choline assay as described in Section III. Each experiment was repeated three times. In each experiment, $15 \mu \mathrm{L}$ of plasma was inserted by pipetting on to the fluidic input. The device was cleaned and reused. A cleaning procedure after each experiment was adopted to avoid cross-contamination.

The cleaning recipe involved subsequent rinse in DI water, IPA, ethanol, and nitrogen blow-dry. Raw data for different concentrations of choline in plasma is shown in Fig. 5(b). For each experiment, data from photodiodes in the same microfluidic channel were averaged and fitted using a second order exponential model. Reaction rates were estimated on the fitted signal. Increased concentrations of the substrate created an increased reaction rate. The quick filling time demonstrated by data in Section IV was essential for an accurate estimation of the initial reaction rate.

Fig. 5(c) presents the calculated initial reaction rate versus the plasma choline concentration. For each data point, data is presented as average and standard deviation over three repeats. Experimental data showed a linear behavior $\left(\mathrm{R}^{2} \geq 0.97\right)$. Relevant metrics illustrated in Table III were quantified using data in Fig. 5(c). The analytical sensitivity was quantified as the slope of the linear calibration curve. The precision of the platform was estimated to be $17.7 \%$ and was calculated as the average standard deviations of the measures. The resolution and the LOD were measured using the International Union of Pure and Applied Chemistry (IUPAC) definition [42] and were found to be $1.6 \mu \mathrm{M}$ and $3.2 \mu \mathrm{M}$, respectively. For the example we present, choline in human plasma, the physiological range is $7-20 \mu \mathrm{M}$ [23], which is sufficiently greater than the LOD to be readily measurable.

The microsystem can quantify choline with concentrations up to one thousand times smaller than those of glucose, cholesterol, and lactate in blood. Even so, operating at this level, the precision of this platform is comparable to the precision of commercial glucose meters ranging from $15 \%$ to $20 \%$ [43]. The test time (5 min) and sample volume $(15 \mu \mathrm{l})$ are also similar to other commercial POC devices measuring cholesterol [44] or lactate [45].

\section{CONClusions}

We demonstrate a fast, low cost and readily implemented multi-channel CMOS-based metabolite sensor. A novel and mass-manufacturable method for packaging and monolithically integrating microfluidics at reasonable cost with integrated circuits for use in POC applications was developed. The results were achieved using a novel doublemoulding approach to manufacture.

The physical characteristics of the fabricated fluidics were characterised, including the channel profile, mechanical strength, optical spectra, and fluid flow in order to verify fast and accurate assay capability. A proof-of-concept device integrated four passive microchannels on top of a CMOS optical sensor array, enabling multiple measurement channels to be made. When used to measure choline, which is only present in small concentrations in human blood, the LOD was found to be $3.2 \mu \mathrm{M}$, delivering a considerable improvement on existing technology, and making POC measurement of metabolites such as choline routinely possible.

The technique is highly versatile and can be used to integrate custom fluidic structures for a range of fluidics-on-integrated circuit applications, including fluidics on CCD cameras. More-than-Moore applications for CMOS technology create new opportunities to further reduce the detection limits of 
POC systems and increase the number of biomarker measurable in a single test. Future opportunities lie in wireless healthcare technology to deliver disposable cartridges measuring multiple biomarkers for early detection of multiple diseases in minutes from a single drop of blood on a handheld device.

\section{ACKNOWLEDGEMENTS}

The authors would like to thank the James Watt Nanofabrication Centre (JWNC), University of Glasgow, and its staff for providing support for the fabrication of the hybrid microfluidics-CMOS device. The research was supported by UKRI grants EP/T00097X and EP/K021966.

\section{REFERENCES}

[1] K. Chakrabarty, R. B. Fair, and J. Zeng, "Design tools for digital microfluidic biochips: Toward functional diversification and more than Moore," IEEE Trans. Comput. Des. Integr. Circuits Syst., vol. 29, no. 7, pp. 1001-1017, Jul. 2010.

[2] Y. Song et al., "Multiplexed volumetric bar-chart chip for point-of-care diagnostics," Nat. Commun. 2012 31, vol. 3, no. 1, pp. 1-9, Dec. 2012.

[3] B. Zethelius et al., "Use of Multiple Biomarkers to Improve the Prediction of Death from Cardiovascular Causes" vol. 358, no. 20, pp. 2107-2116, Jul. 2009.

[4] S. B. Patil, V. F. Annese, and D. R. S. Cumming, "Commercial Aspects of Biosensors for Diagnostics and Environmental Monitoring," in Advances in Nanosensors for Biological and Environmental Analysis, Elsevier, 2019, pp. 133-142.

[5] T. Datta-Chaudhuri, E. Smela, and P. A. Abshire, "System-on-Chip Considerations for Heterogeneous Integration of CMOS and Fluidic Bio-Interfaces," IEEE Trans. Biomed. Circuits Syst., vol. 10, no. 6, pp. 1129-1142, Dec. 2016

[6] M. S. Andrianova et al., "CMOS-compatible biosensor for L-carnitine detection," Biosens. Bioelectron., vol. 119, pp. 48-54, Nov. 2018.

[7] Y. W. Chang et al., "A CMOS-compatible optical biosensing system based on visible absorption spectroscopy," IEEE Conf. Electron Devices Solid-State Circuits 2007, EDSSC 2007, pp. 1099-1102, 2007.

[8] K. R. Sreejith et al., "Digital polymerase chain reaction technology recent advances and future perspectives," Lab Chip, vol. 18, no. 24, pp. 3717-3732, Dec. 2018.

[9] L. Cao et al., "A Portable Digital Loop-Mediated Isothermal Amplification Platform Based on Microgel Array and Hand-Held Reader," ACS Sensors, vol. 6, no. 10, pp. 3564-3574, Oct. 2021,

[10] Z. Li et al., "Fully integrated microfluidic devices for qualitative, quantitative and digital nucleic acids testing at point of care," Biosens. Bioelectron., vol. 177, p. 112952, Apr. 2021.

[11] Y. Huang and A. J. Mason, "Lab-on-CMOS integration of microfluidics and electrochemical sensors," Lab Chip, vol. 13, no. 19, pp. 3929-3934, 2013.

[12] F. J. Blanco et al., "Microfluidic-optical integrated CMOS compatible devices for label-free biochemical sensing," J. Micromechanics Microengineering, vol. 16, no. 5, pp. 1006-1016, 2006.

[13] A. Wu et al. , "Modular integration of electronics and microfluidic systems using flexible printed circuit boards," Lab Chip, vol. 10, no. 4, pp. 519-521, 2010.

[14] C. P. Hsu, P. C. Chen, and Y. L. Wang, "A novel packaging technology for disposable FET-based biosensors with microfluidic channels," 2017 IEEE 12th Int. Conf. Nano/Micro Eng. Mol. Syst. NEMS 2017, pp. 375378, 2017.

[15] D. Welch and J. B. Christen, "Seamless integration of CMOS and microfluidics using flip chip bonding," J. Micromechanics Microengineering, vol. 23, no. 3, 2013.

[16] M. Muluneh and D. Issadore, "A multi-scale PDMS fabrication strategy to bridge the size mismatch between integrated circuits and microfluidics," Lab Chip, vol. 14, no. 23, pp. 4552-4558, 2014.

[17] E. Ghafar-Zadeh, M. Sawan, and D. Therriault, "Novel direct-write CMOS-based laboratory-on-chip: Design, assembly and experimental results," Sensors Actuators, A Phys., vol. 134, no. 1, pp. 27-36, 2007.

[18] H. Lee et al. "Integrated cell manipulation system - CMOS/microfluidic hybrid," Lab Chip, vol. 7, no. 3, pp. 331-337, 2007.
[19] S. M. Khan et al. "CMOS Enabled Microfluidic Systems for Healthcare Based Applications," Adv. Mater., vol. 30, no. 16, pp. 1-26, 2018.

[20] B. Zhang et al. "Flexible packaging of solid-state integrated circuit chips with elastomeric microfluidics," Sci. Rep., vol. 3, pp. 1-8, 2013.

[21] V. Ortseifen et al. "Microfluidics for Biotechnology: Bridging Gaps to Foster Microfluidic Applications," Frontiers in Bioengineering and Biotechnology, vol. 8. Frontiers Media S.A., p. 1324, Nov. 13, 2020.

[22] S. Noijen et al., "Prediction of the epoxy moulding compound aging effect on package reliability," Microelectron. Reliab., vol. 50, no. 7, pp. 917-922, Jul. 2010.

[23] B. M. Pitkin RM, Allen LH, Bailey LB, "Dietary Reference Intakes for Thiamin, Riboflavin, Niacin, Vitamin B6, Folate, Vitamin B12, Pantothenic Acid, Biotin, and Choline," Washington (DC): National Academies Press (US); 1998., 1998.

[24] P. P. Liu et al., "Discovery of Metabolite Biomarkers for Acute Ischemic Stroke Progression,” J. Proteome Res., vol. 16, no. 2, pp. 773779, Feb. 2017.

[25] Z. Y. Liu et al., "Serum choline is associated with hepatocellular carcinoma survival: A prospective cohort study," Nutrition and Metabolism, vol. 17, no. 1. BioMed Central Ltd., Mar. 30, 2020.

[26] P. Rahimi and Y. Joseph, "Enzyme-based biosensors for choline analysis: A review," TrAC Trends Anal. Chem., vol. 110, pp. 367-374, Jan. 2019

[27] W. Wang et al., "LC-MS/MS Analysis of Choline Compounds in Japanese-Cultivated Vegetables and Fruits," Foods, vol. 9, no. 8, Aug. 2020.

[28] M. A. Al-Rawhani et al., "Multimodal Integrated Sensor Platform for Rapid Biomarker Detection.," IEEE Trans. Biomed. Eng., Jun. 2019.

[29] Annese, V.F., et al. A monolithic single-chip point-of-care platform for metabolomic prostate cancer detection. Microsyst Nanoeng 7, 21. 2021.

[30] V. F. Annese et al., "The Multicorder: A Handheld Multimodal Metabolomics-on-CMOS Sensing Platform," 2019 IEEE 8th International Workshop on Advances in Sensors and Interfaces (IWASI), 2019, pp. 130-135.

[31] C. Hu et al., "Disposable Paper-on-CMOS Platform for Real-Time Simultaneous Detection of Metabolites," in IEEE Transactions on Biomedical Engineering, vol. 67, no. 9, pp. 2417-2426, Sept. 2020.

[32] H. Bruus, Theoretical microfluidics. Oxford University Press, 2008.

[33] "DDBST - DDBST GmbH." http://www.ddbst.com/ (accessed Feb. 17, 2020).

[34] "Viscosity of Blood Plasma and Serum." https://www.rheosense.com/application/viscosity-of-blood-plasma-andserum (accessed Feb. 17, 2020).

[35] S. Lewin, "Blood Serum Surface Tension and its Potential," Br. J. Haematol., vol. 22, no. 5, pp. 561-566, May 1972.

[36] E. Hrnčír and J. Rosina1, "Surface Tension of Blood," 1997.

[37] T. Trantidou et al., "Hydrophilic surface modification of pdms for droplet microfluidics using a simple, quick, and robust method via pva deposition," Microsystems Nanoeng., vol. 3, no. April 2016, 2017.

[38] A. Syakur, Hermawan, and H. Sutanto, "Determination of hydrophobic contact angle of epoxy resin compound silicon rubber and silica," in International Conference on Electrical Engineering, Computer Science and Informatics (EECSI), 2016, vol. 3, no. 1, p. 012025.

[39] R. Seghir and S. Arscott, "Sensors and Actuators A : Physical Extended PDMS stiffness range for flexible systems," Sensors Actuators A. Phys., vol. 230, pp. 33-39, 2015.

[40] "Thorlabs - LED490L $490 \mathrm{~nm}$ LED with a Glass Lens, $3 \mathrm{~mW}$, TO-18." https://www.thorlabs.com/thorproduct.cfm?partnumber=LED490L (accessed Dec. 13, 2021)

[41] C. Accarino et al., "Noise characteristics with CMOS sensor array scaling," Meas. J. Int. Meas. Confed., vol. 152, 2020.

[42] N. Bhalla, P. Jolly, N. Formisano, and P. Estrela, "Introduction to biosensors," Essays Biochem., pp. 60-61, 2016.

[43] A. St John and C. P. Price, "Existing and Emerging Technologies for Point-of-Care Testing.," Clin. Biochem. Rev., vol. 35, no. 3, pp. 155-67, Aug. 2014, Accessed: Jan. 27, 2020. [Online]. Available: http://www.ncbi.nlm.nih.gov/pubmed/25336761.

[44] "Cholestech LDX Analyzer | Abbott Point of Care Testing." https://www.globalpointofcare.abbott/en/product-details/cholestech-ldxsystem.html (accessed Oct. 01, 2021).

[45] "i-STAT System | Abbott Point of Care." https://www.pointofcare.abbott/int/en/offerings/istat (accessed Jan. 30, 2020). 